\title{
Calcium channel blockers in acute myocardial infarction and unstable angina: an overview
}

\author{
Peter H Held, Salim Yusuf, Curt D Furberg
}

\begin{abstract}
Objective-To assess the effects of calcium channel blockers on development of infarcts, reinfarction, and mortality.

Design-A systematic overview of all randomised trials of calcium channel blockers in myocardial infarction and unstable angina.

Patients - 19000 Patients in 28 randomised trials.

Results-In the trials of myocardial infarction 873 deaths occurred among 8870 patients randomised to active treatment compared with 825 deaths among 8889 control patients (odds ratio of $1.06,95 \%$ confidence interval of 0.96 to $1 \cdot 18$ ). There was no evidence of a beneficial effect on development and size of infarcts or rate of reinfarction. The results were similar in short term trials in which treatment was confined to the acute phase and those in which treatment was started some weeks later and continued for a year or two. There was no evidence of heterogeneity among different calcium channel blockers in their effects on any end point. The results were similar in the unstable angina trials (110 out of 561 patients treated with calcium channel blocker compared with 104 out of 548 controls developed a myocardial infarction; 14 out of 591 treated compared with nine out of 578 controls died).
\end{abstract}

Conclusions-Calcium channel blockers do not reduce the risk of initial or recurrent infarction or death when given routinely to patients with acute myocardial infarction or unstable angina.

\section{Introduction}

Calcium channel blockers have been shown in animal studies to reduce the extent of myocardial necrosis and to preserve ventricular function after experimental occlusion of the coronary artery. ${ }^{\prime}$ These effects are probably mediated by a reduction in afterload leading to reduced oxygen consumption, by increased coronary blood flow due to coronary vasodilatation, and by prevention of myocardial cell damage through decreasing overload of intracellular calcium during ischaemia. These experimental trials provided a basis for evaluating whether calcium channel blockers reduce the incidence of complications and death in patients with unstable angina and in those with suspected or established myocardial infarction. Although several randomised trials have been conducted, most studies have been too small to have sufficient statistical power to detect moderately beneficial or harmful effects (for example, $15 \%$ or $20 \%$ ), whereas several have been sufficiently large to detect or exclude fairly large effects (for example, $30 \%$ to $40 \%$ ). In such cases a more reliable estimate of overall treatment effect can be obtained by a systematic overview of all relevant trials. Thus our main aim was to review systematically the data from all randomised controlled trials of calcium channel blockers, regarding occurrence of first myocardial infarction, infarct size, prevention of reinfarction, and death in patients with unstable angina and acute myocardial infarction.

Although there are substantial similarities in their mechanism of action, the various calcium channel blockers differ somewhat in their ancillary properties. ${ }^{2}$ Some agents, such as nifedipine, can cause a tachycardia, whereas diltiazem and verapamil reduce heart rate, actions that might influence their clinical effects. In this overview, we present the effects of treatment for each outcome of interest; firstly, for the overall data, secondly, by each agent, and, thirdly, by category of agents that share some common ancillary properties.

\section{Methods}

The methods are similar to those described and used previously. ${ }^{3}$ The intent was to obtain data on mortality and, when relevant, initial or recurrent myocardial infarction and infarct size from each completed, published or unpublished, randomised controlled trial of any calcium channel blocking agent in patients with suspected or definite myocardial infarction or in patients with unstable angina. Our method was to scan the journals, both by a formal computer aided search and by an informal search for studies that were known to ourselves or to coworkers; to scan the reference lists of the published papers; and to inquire about uncompleted or completed but unpublished trials from colleagues. When possible if the data thus obtained did not include all randomised patients or did not include certain end points (such as reinfarction) of particular interest we sought additional details by correspondence, generally with the principal investigators. Data on outcome from the different trials were combined by the Mantel-Haenszel method. ${ }^{3}$ The analyses were performed separately for each agent, for the short term and long term trials separately, and for the trials in total.

The underlying principle was that patients allocated to active treatment in one trial were compared directly only with those allocated to control in the same trial and not with patients in any other trial. Therefore, differences in the characteristics of patients between trials did not matter as long as the patients were broadly similar (for example, suspected acute myocardial infarction). For each trial the number of events observed in the treated group was contrasted with the number that would have been expected if treatment had no effect. If treatment was of no benefit differences between the observed and the expected events would differ only randomly from zero. A beneficial treatment effect with fewer events in the actively treated group would result in a negative value for observed minus expected, while a harmful treatment would result in a positive value. In a single trial this tendency might be obscured by chance, but when several individual trials 
are combined to give a grand total of all the values such chance variations tend to be averaged out.

Determination of the effects of treatment from the grand total assumes that information is available from all trials of an unbiased subsample. It does not, however, assume that the treatment effect is the same in different trials but rather that this effect will tend to be in the same direction in most trials. The variance of the grand total is the sum of the variances of the separate values for observed minus expected. The overall effect of treatment may be tested by the formula $\mathrm{Z}=\mathrm{GT} / \sqrt{\mathrm{VT}}$, where $\mathrm{Z}=1.96$ indicates a two tailed $\mathrm{p}$ value of $0 \cdot 05, \mathrm{GT}=$ grand total, and $\mathrm{VT}=$ variance of grand total. An estimate of the odds of an event (typical odds ratio) in treated patients compared with controls is given by the formula $\exp (\mathrm{GT} / \mathrm{VT})$, with approximate $95 \%$ confidence intervals by $\exp (\mathrm{GT} / \mathrm{VT} \pm$ $1 \cdot 96 / \sqrt{\text { VT }})$. Tests for interaction between any two categories of trials follow naturally: if $S_{1}, C_{1}, S_{2}$, and $C_{2}$ are the respective sums of the values for observed minus expected $(\mathrm{O}-\mathrm{E})$ and variance for two categories of trial that are to be contrasted with each other the test is whether $S_{1} / C_{1}$ (with variance $1 / C_{1}$ ) differs significantly from $S_{2} / C_{2}$ (with variance $1 / C_{2}$ ) so the approximate $\chi^{2}$ test statistic for such an interaction is $\left(\mathrm{S}_{1} / \mathrm{C}_{1}-\mathrm{S}_{2} / \mathrm{C}_{2}\right)^{2} /\left(1 / \mathrm{C}_{1}+1 / \mathrm{C}_{2}\right)$. An overall $\chi^{2}$ test of heterogeneity can be calculated by $\Sigma\left[\left(\mathrm{O}_{1}-\mathrm{E}_{1}\right)^{2} / \mathrm{V}_{1}\right]-$ $\left[\Sigma\left(\mathrm{O}_{1}-\mathrm{E}_{1}\right)\right]^{2} / \Sigma \mathrm{V}_{1}$, with degrees of freedom equalling 1 less than the number of non-zero variances. ${ }^{3}$

\section{Results}

DESCRIPTION OF TRIALS

Twenty two randomised controlled trials of calcium channel blockers in myocardial infarction were identified by January 1989 (table I). ${ }^{425}$ In 17 of these studies treatment was started within a few hours after onset of symptoms and continued short term ${ }^{4-20}$; in two trials treatment was started early and continued long term $^{2122}$; and in three trials treatment was begun some

TABLE I-Design of trials evaluating calcium channel blockers in acute myocardial infarction

\begin{tabular}{|c|c|c|c|c|c|c|}
\hline $\begin{array}{l}\text { Treatment and } \\
\text { authors }\end{array}$ & Dosage & Criteria for exclusion & $\begin{array}{l}\text { Time of starting } \\
\text { treatment }\end{array}$ & $\begin{array}{l}\text { Duration of } \\
\text { treatment }\end{array}$ & Primary end point & Design \\
\hline \multicolumn{7}{|c|}{ Acute, short term studies } \\
\hline \multicolumn{4}{|c|}{ Pulmonary capillary wedge pressure $<20 \mathrm{~h}$ (mean $8 \mathrm{~h}$ ) $48 \mathrm{~h}$} & $48 \mathrm{~h}$ & & Open \\
\hline Crea $e t a l^{\varsigma}$ & $\begin{array}{l}4 \times 10 \mathrm{mg} \mathrm{IV} \text { and } \\
4 \times 80 \mathrm{mg} \text { orally }\end{array}$ & $\begin{array}{l}\text { Systolic blood pressure }<90 \mathrm{~mm} \mathrm{Hg} \text {, } \\
\text { heart rate }<55 / \mathrm{min}, \\
\text { atrioventricular block, congestive } \\
\text { heart failure }\end{array}$ & $<12 \mathrm{~h}$ & 10 Days & $\begin{array}{l}\text { Angina and } \\
\text { reinfarction }\end{array}$ & Single blind \\
\hline $\begin{array}{l}\text { Nifedipine: } \\
\text { Sirnes } \text { et } a l^{6}\end{array}$ & $5 \times 10 \mathrm{mg}$ & Congestive heart failure, & Mean $5 \cdot 2 \mathrm{~h}$ & 6 Weeks & Infarct size & Double blind \\
\hline Muller $e t a l^{7}$ & $4 \times 20 \mathrm{mg}$ & $\begin{array}{l}\text { Systolic blood pressure }<110 \mathrm{~mm} \\
\text { Hg, shock }\end{array}$ & $<6 \mathrm{~h}($ mean $4.6 \mathrm{~h})$ & 2 Weeks & Infarct development & Double blind \\
\hline Gordon et $a l^{8}$ & $4 \times 10 \mathrm{mg}$ & $\begin{array}{l}\text { Systolic blood pressure }<90 \mathrm{~mm} \mathrm{Hg} \text {, } \\
\text { arrhythmias }\end{array}$ & $<12 \mathrm{~h}$ & $24 \mathrm{~h}$ & $\begin{array}{l}\text { Central } \\
\text { haemodynamics }\end{array}$ & Single blind \\
\hline Gottlieb et al ${ }^{9}$ & $40-120 \mathrm{mg}$ total/day & $\begin{array}{l}\text { Ejection fraction }<35 \% \text {, systolic } \\
\text { blood pressure }<100 \mathrm{~mm} \mathrm{Hg} \text {, } \\
\text { congestive heart failure }\end{array}$ & $<12 \mathrm{~h}($ mean $8 \mathrm{~h})$ & 6 Weeks & $\begin{array}{l}\text { Left ventricular } \\
\text { function plus infarct } \\
\text { size }\end{array}$ & Double blind \\
\hline Eisenberg et $a l^{10}$ & $4 \times 20 \mathrm{mg}$ & $\begin{array}{l}\text { Q-wave infarction, congestive heart } \\
\text { failure }\end{array}$ & Infarct diagnosis & 14 Days & Chest pain & Double blind \\
\hline Branagan $e t a l^{\prime \prime}$ & $4 \times 10 \mathrm{mg}$ & $\begin{array}{l}\text { Systolic blood pressure }<85 \mathrm{~mm} \mathrm{Hg} \text {, } \\
\text { heart rate }<60 / \mathrm{min}, \text { congestive } \\
\text { heart failure }\end{array}$ & $<6 \mathrm{~h}($ mean $3.4 \mathrm{~h})$ & $48 \mathrm{~h}$ & Infarct size, mortality & Double blind \\
\hline Loogna et $a l^{12}$ & $4 \times 10 \mathrm{mg}$ & Non-cardiac disease & $<6 \mathrm{~h}($ mean $3.4 \mathrm{~h})$ & Not known & Infarct size & Double blind \\
\hline Wilcox et al ${ }^{13}$ & $4 \times 10 \mathrm{mg}$ & $\begin{array}{l}\text { Systolic blood pressure }<100 \mathrm{~mm} \\
\mathrm{Hg}, \text { heart rate }>120 / \mathrm{min}, \\
\text { congestive heart failure }\end{array}$ & $<24 \mathrm{~h}(68 \%<8 \mathrm{~h})$ & 4 Weeks & Mortality & Double blind \\
\hline Walker $e t$ al ${ }^{14}$ & $6 \times 10 \mathrm{mg}$ & Systolic blood pressure $<85 \mathrm{~mm} \mathrm{Hg}$ & $<6 \mathrm{~h}$ & $48 \mathrm{~h}$ & Infarct size & Double blind \\
\hline & $6 \times 20 \mathrm{mg}$ & $\begin{array}{l}\text { Systolic blood pressure }<110 \mathrm{~mm} \\
\mathrm{Hg} \text {, shock }\end{array}$ & $\begin{array}{l}<18 \mathrm{~h}(\text { mean } \\
9 \cdot 6 \mathrm{~h})\end{array}$ & 1 Week & $\begin{array}{l}\text { Myocardial } \\
\text { metabolism }\end{array}$ & Single blind \\
\hline Erbel $e t a l^{16}$ & $\begin{array}{l}0.2 \mathrm{mg} \text { Intracoronary } \\
\text { plus } 3 \times 20 \mathrm{mg}\end{array}$ & Contraindications to thrombolysis & $<6 \mathrm{~h}$ & Hospital stay & Infarct size & Double blind \\
\hline \multicolumn{7}{|l|}{ Diltiazem: } \\
\hline Gibson et al ${ }^{17}$ & $4 \times 30 \mathrm{mg}$ & $\begin{array}{l}\text { Systolic blood pressure }<100 \mathrm{~mm} \\
\mathrm{Hg}, \mathrm{Q} \text { wave myocardial } \\
\text { infarction, congestive heart } \\
\text { failure, heart rate }<50 / \mathrm{min}\end{array}$ & $24-72 \mathrm{~h}$ & 2 Weeks & Reinfarction & Double blind \\
\hline Zannad et al ${ }^{18}$ & $\begin{array}{l}10-20 \mathrm{mg} / \mathrm{h} \mathrm{IV}, 4 \times 60 \\
\mathrm{mg} \text { orally }\end{array}$ & $\begin{array}{l}\text { Atrioventricular block, congestive } \\
\text { heart failure }\end{array}$ & $<6 \mathrm{~h}$ & 3 Weeks & Infarct size & Double blind \\
\hline $\begin{array}{l}\text { Machecourt } \\
\text { et al }\end{array}$ & $\begin{array}{l}360 \mathrm{mg} \text { IV/24 h plus } \\
360 \mathrm{mg} / 24 \mathrm{~h} \text { orally }\end{array}$ & $\begin{array}{l}\text { Atrioventricular block, shock, } \\
\text { congestive heart failure }\end{array}$ & $<6 \mathrm{~h}$ & 3 Weeks & Infarct size & Single blind \\
\hline \multicolumn{6}{|l|}{ Tiapamil: } & \\
\hline & $25 \mathrm{mg} / \mathrm{kg} / \mathrm{min}$ IV & $\begin{array}{l}\text { atrioventricular block, congestive } \\
\text { heart failure }\end{array}$ & 7 & & haemodynamics & \\
\hline \multicolumn{7}{|c|}{ Acute and long term studies } \\
\hline \multirow{2}{*}{$\begin{array}{l}\text { Verapamil: } \\
\text { Danish study }\end{array}$} & & & & & & \\
\hline & $\begin{array}{l}0.1 \mathrm{mg} / \mathrm{kg} \mathrm{IV} \text { plus } \\
3 \times 120 \mathrm{mg} \text { orally }\end{array}$ & $\begin{array}{l}\text { Systolic blood pressure }<90 \mathrm{~mm} \mathrm{Hg} \text {, } \\
\text { heart rate }<45 / \mathrm{min}, \text { congestive } \\
\text { heart failure }\end{array}$ & $85 \%<24 \mathrm{~h}$ & 6 Months & $\begin{array}{l}\text { Mortality plus } \\
\text { reinfarction }\end{array}$ & Double blind \\
\hline $\begin{array}{l}\text { Nifedine: } \\
\text { SPRINT-II }\end{array}$ & $3 \times 20 \mathrm{mg}$ & Systolic blood pressure $<90 \mathrm{~mm} \mathrm{Hg}$ & $<48 \mathrm{~h}$ & 6 Months & Mortality & Double blind \\
\hline \multicolumn{7}{|c|}{ Long term studies } \\
\hline $\begin{array}{l}\text { Lidoflazine: } \\
\text { MI Study } \\
\text { Group }^{23}\end{array}$ & $3-4 \times 60 \mathrm{mg}$ & $\begin{array}{l}\text { Severe hypertension, } \\
\text { atrioventricular block, congestive } \\
\text { heart failure }\end{array}$ & 2 Months & 5 Years & Mortality & Open \\
\hline $\begin{array}{l}\text { Nifedipine: } \\
\text { SPRINT-I }{ }^{24}\end{array}$ & $3 \times 10 \mathrm{mg}$ & $\begin{array}{l}\text { Systolic blood pressure }<90 \mathrm{~mm} \mathrm{Hg} \text {, } \\
\text { atrioventricular block, congestive } \\
\text { heart failure }\end{array}$ & 7-21 Days & 1 Year & Mortality & Double blind \\
\hline $\begin{array}{l}\text { Diltiazem: } \\
\text { MDPIT }^{25}\end{array}$ & $240 \mathrm{mg} / 24 \mathrm{~h}$ & $\begin{array}{l}\text { Hypotension, atrioventricular } \\
\text { block, heart rate }<50 / \mathrm{min} \text {, shock }\end{array}$ & 3-15 Days & $\begin{array}{l}\text { 1-5 Years } \\
\text { (mean 25 } \\
\text { months) }\end{array}$ & Mortality & Double blind \\
\hline
\end{tabular}




\begin{tabular}{|c|c|c|c|c|c|c|}
\hline \multirow[b]{2}{*}{ Trial } & \multirow[b]{2}{*}{ Treatment } & \multirow[b]{2}{*}{$\begin{array}{c}\text { Duration } \\
\text { of } \\
\text { treatment }\end{array}$} & \multicolumn{2}{|c|}{$\begin{array}{c}\text { No who developed infarct/ } \\
\text { No randomised }\end{array}$} & \multicolumn{2}{|c|}{ No of deaths } \\
\hline & & & $\begin{array}{l}\text { Allocated to } \\
\text { calcium } \\
\text { blocker }\end{array}$ & $\begin{array}{c}\text { Allocated } \\
\text { to } \\
\text { control }\end{array}$ & $\begin{array}{l}\text { Allocated to } \\
\text { calcium } \\
\text { blocker }\end{array}$ & $\begin{array}{c}\text { Allocated } \\
\text { to } \\
\text { control }\end{array}$ \\
\hline \multirow{4}{*}{$\begin{array}{l}\text { Placebo as control: } \\
\text { Gerstenblith } \text { et }^{2 l^{26}} \\
\text { HINT }^{27} \\
\text { Hagemeijer } \text { et }_{\text {al }}{ }^{28}\end{array}$} & & & & & & \\
\hline & Nifedipine & 4 months & $11 / 68$ & $12 / 70$ & $7 / 68$ & $5 / 70$ \\
\hline & Nifedipine & $48 \mathrm{~h}$ & $78 / 341$ & $77 / 327$ & $1 / 341$ & $2 / 327$ \\
\hline & Diltiazem & $48 \mathrm{~h}$ & NA & NA & $0 / 30$ & $0 / 30$ \\
\hline Subtotal & & & $89 / 409$ & $89 / 397$ & $8 / 439$ & $7 / 427$ \\
\hline \multicolumn{7}{|c|}{ Standard treatment as control: } \\
\hline Muller et $a l^{29}$ & Nifedipine & $48 \mathrm{~h}$ & $13 / 68$ & $10 / 65$ & $4 / 68$ & $0 / 65$ \\
\hline Theroux et $a l^{30}$ & Diltiazem & 5.1 months & $5 / 50$ & $4 / 50$ & $2 / 50$ & $2 / 50$ \\
\hline André-Fouet $e t a l^{31}$ & Diltiazem & $48 \mathrm{~h}$ & $3 / 34$ & $1 / 36$ & $0 / 34$ & $0 / 36$ \\
\hline Subtotal & & & $21 / 152$ &, $15 / 151$ & $6 / 152$ & $2 / 151$ \\
\hline Total & & & $110 / 561$ & $104 / 548$ & $14 / 591$ & $9 / 578$ \\
\hline
\end{tabular}

$\mathrm{NA}=$ not available.

days to weeks after the infarction and continued for one to five years. ${ }^{23-25}$ About 18000 patients were studied in total, and the number of patients in the individual trials varied from about 20 to 4500 . Verapamil was evaluated in three trials (with a total of 3500 patients), nifedipine in 13 trials ( 9700 patients), diltiazem in four trials (3100 patients), tiapamil in one trial (32 patients), and lidoflazine in one trial ( 1800 patients). Most of the trials of early treatment comprised patients with suspected acute myocardial infarction, but two trials enrolled only patients with confirmed infarction. ${ }^{10}{ }^{17}$ All patients in the long term studies were survivors of an acute myocardial infarction..$^{23-25}$ The exclusion criteria were fairly similar in the different trials (table I). Typically patients were excluded if systolic blood pressure was low $(85-110 \mathrm{~mm} \mathrm{Hg})$ or, in some studies, very high; if heart rate was low ( $45-60$ beats $/ \mathrm{min})$ or high (110-120 beats/min); if severe heart failure or shock was present; or if the patient had atrioventricular block.

The dose of study drug and the duration of treatment varied. In six of the acute trials treatment was started intravenously, ${ }^{4518-21}$ while the other trials used only oral drugs. The daily oral dose varied, the ranges being $320-360 \mathrm{mg}$ verapamil, $30-120 \mathrm{mg}$ nifedipine, $120-360 \mathrm{mg}$ diltiazem, and $180-240 \mathrm{mg}$ lidoflazine. The scheduled study period varied from between 24 hours and six weeks in the acute short term trials, was six months in the combined acute and long term trials, and varied between one and five years in the long term trials. All but six trials were double blind; two were open and four were single blind (table I).

Six trials with a total of 1100 patients with unstable angina were identified (table II). ${ }^{26-31}$ Three of the trials used propranolol as control..$^{29-31}$ Two small randomised trials of verapamil were excluded because of their crossover design ${ }^{32}{ }^{33}$; only one myocardial infarction or death occurred in these trials and this event would not alter the conclusions if it was included in the present overview. Most patients were entered into the three nifedipine trials, while diltiazem was studied in three small trials. Patients were generally included if they had angina at rest or if they had frequently recurring angina, abnormalities on an electrocardiogram, and a history of coronary disease. Four trials were double blind $^{26-29}$ and two were single blind ${ }^{3031}$; the duration of treatment varied from 48 hours to four months.

\section{ACUTE MYOCARDIAL INFARCTION TRIALS \\ Mortality}

Complete mortality data by allocated treatment were available for 18 of the 22 trials (table III). No data were available for one fairly small trial. ${ }^{12}$ In another trial mortality data were not available for the $139(68$ placebo and 71 nifedipine) patients who did not develop a myocardial infarction and for another 69 (29 placebo and 40 nifedipine) patients who were treated with streptokinase or were withdrawn after randomisation. ${ }^{14}$ In the long term lidoflazine trial follow up data were missing on 25 of the randomised 1817 patients (allocation unknown). ${ }^{23}$ In the Danish study $^{21}$ only patients who developed confirmed infarction were treated after seven days. Therefore, no data are available beyond this period for 2011 of the 3447 patients who initially entered the trial. One trial was stoppęd early because of a trend towards more deaths in the active treatment group. ${ }^{22}$

In three trials mortality was lower in the group allocated to receive calcium channel blockers compared with those allocated to the control group; in five trials no difference was found; and in 13 trials mortality was higher. None of these individual differences was significant. Overall, there were 873 deaths in 8870 patients $(9 \cdot 8 \%)$ allocated to active treatment compared with 825 deaths among 8889 controls $(9 \cdot 3 \%)$ during the scheduled trial period (typical odds of death was increased by $6 \%, 95 \%$ confidence interval $-4 \%$ to $18 \%)$

Whether treatment was started early and continued short term or long term or was started later and continued long term did not materially influence the outcome (table III, figure). A small but non-significant increase in mortality in patients allocated calcium channel blockers was observed in all three categories of trials.

TABLE III - Mortality of patients during trial period in trials of calcium channel blockers

\begin{tabular}{|c|c|c|c|c|}
\hline \multirow[b]{2}{*}{ Trial } & \multicolumn{2}{|c|}{ No of deaths/No randomised } & \multirow{2}{*}{$\begin{array}{c}\text { Observed } \\
\text { minus } \\
\text { expected }\end{array}$} & \multirow[b]{2}{*}{ Variance } \\
\hline & $\begin{array}{l}\text { Allocated to } \\
\text { calcium blocker }\end{array}$ & $\begin{array}{l}\text { Allocated to } \\
\text { control }\end{array}$ & & \\
\hline \multicolumn{5}{|c|}{ Acute short term trials } \\
\hline Bussman et $a l^{4 \star}$ & $0 / 29$ & $0 / 25$ & $0 \cdot 0$ & $0 \cdot 0$ \\
\hline Crea et al & $0 / 8$ & $2 / 9$ & -0.9 & 0.5 \\
\hline Sirnes et $a l^{\circ}$ & $10 / 112$ & $10 / 115$ & $0 \cdot 1$ & $4 \cdot 6$ \\
\hline Muller et al & $7 / 93$ & $2 / 88$ & $2 \cdot 4$ & $2 \cdot 0$ \\
\hline Gordon et $a l^{8 \star}$ & $0 / 13$ & $0 / 13$ & $0 \cdot 0$ & $0 \cdot 0$ \\
\hline Gottlieb et $a l^{9}$ & $4 / 64$ & $4 / 68$ & $0 \cdot 1$ & 1.9 \\
\hline Eisenberg et $a l^{10 \star}$ & $0 / 25$ & $0 / 25$ & $0 \cdot 0$ & $0 \cdot 0$ \\
\hline Branagan et al $l^{11 \star}$ & $7 / 60$ & $5 / 68$ & $1 \cdot 0$ & $2 \cdot 7$ \\
\hline Loogna et al ${ }^{12}$ & $\mathrm{NA} / 23$ & $\mathrm{NA} / 34$ & NA & NA \\
\hline Wilcox et $a l^{13}$ & $150 / 2240$ & $141 / 2251$ & 4.9 & $68 \cdot 1$ \\
\hline Walker et al ${ }^{14} \dagger$ & $7 / 106$ & $7 / 120$ & 0.4 & $3 \cdot 3$ \\
\hline Jaffe et al ${ }^{15}$ & $1 / 13$ & $0 / 9$ & $0 \cdot 4$ & $0 \cdot 2$ \\
\hline Erbel et al $l^{10}$ & $10 / 74$ & $6 / 75$ & $2 \cdot 1$ & $3 \cdot 6$ \\
\hline Gibson et al ${ }^{17}$ & $11 / 287$ & $9 / 289$ & $1 \cdot 0$ & $4 \cdot 8$ \\
\hline Zannad et al l $^{18}$ & $1 / 17$ & $1 / 17$ & $0 \cdot 0$ & 0.5 \\
\hline Machecourt et al l9 $^{19}$ & $2 / 38$ & $4 / 37$ & $-1 \cdot 0$ & 1.4 \\
\hline Eichler $e t a l^{20}$ & $0 / 16$ & $0 / 16$ & $0 \cdot 0$ & $0 \cdot 0$ \\
\hline Subtotal & $210 / 3195(6 \cdot 6 \%)$ & $191 / 3225(5 \cdot 9 \%)$ & $9 \cdot 5$ & $93 \cdot 6$ \\
\hline \multicolumn{5}{|c|}{ Acute and long term trials } \\
\hline Danish $^{21} \ddagger$ & $149 / 1729$ & $145 / 1718$ & 1.5 & $67 \cdot 2$ \\
\hline SPRINT-II ${ }^{2 \star}$ & $105 / 680$ & $90 / 678$ & $7 \cdot 4$ & $41 \cdot 8$ \\
\hline Subtotal & $254 / 2409(10 \cdot 5 \%$ & $235 / 2396(9 \cdot 8 \%)$ & 8.9 & $109 \cdot 0$ \\
\hline \multicolumn{5}{|c|}{ Long term trials } \\
\hline MI Study Group ${ }^{23} \mathbb{S}$ & $178 / 904$ & $167 / 888$ & $4 \cdot 0$ & $69 \cdot 7$ \\
\hline SPRINT-I ${ }^{24}$ & $65 / 1130$ & $65 / 1146$ & 0.5 & 30.6 \\
\hline MDPIT $^{25}$ & $166 / 1232$ & $167 / 1234$ & $-0 \cdot 4$ & $72 \cdot 0$ \\
\hline Subtotal & $409 / 3266(12 \cdot 5 \%$ & $399 / 3268(12 \cdot 2 \%)$ & $4 \cdot 1$ & $172 \cdot 3$ \\
\hline Tot & $873 / 8870(9 \cdot 8 \%)$ & $825 / 8889(9 \cdot 3 \%)$ & $23 \cdot 5$ & 374.9 \\
\hline
\end{tabular}

*Additional data obtained through principal investigator.

tNo data available on 208 additional excluded patients (see text)

fOnly 1436 patients with confirmed myocardial infarction were treated beyond seven days. No data are available on remaining patients beyond this period.

period.

Data missing on 25 patients.

Typical odds ratio ( $95 \%$ confidence interval) for acute short term trials $1 \cdot 11(0.90$ to 1.36$)$; for acute and long term trials $1.09(0.90$ to 1.31$)$; and fo long term trials $1.03(0.89$ to $1 \cdot 20)$. Overall typical odds ratio $(95 \%$ confidence interval) $1.06(0.96$ to 1.18$)$. No results or overall excess in mortality significant at $p \leqslant 0.05$. Test for overall heterogeneity among trials within category: acute short term trials $\chi^{2} 7 \cdot 5,11 \mathrm{df}$; acute and long term trials $\chi^{2} 0 \cdot 6,1 \mathrm{df} ;$ long term trials $\chi^{2} 0 \cdot 1,2 \mathrm{df}$

$\mathrm{NA}=$ not available or not known. 


\section{Development of myocardial infarction}

Patients with an initial diagnosis of suspected acute myocardial infarction were included in 17 trials in which treatment was started in most patients within 24 hours after onset of symptoms. The proportion of patients developing a definite infarction was lower in patients allocated to calcium channel blocker compared with controls in seven trials and higher in three trials; no difference was observed in seven trials (table IV). In the seven small trials without a difference all patients developed a myocardial infarction, indicating that only patients with a very high probability of this were included. In total, 3328 of 5426 actively treated patients $(61 \cdot 3 \%)$ developed a myocardial infarction compared with 3364 of the 5431 controls

TABLE IV-Development of infarcts in trials in which treatment with calcium channel blockers was started within 24 hours

\begin{tabular}{|c|c|c|c|c|}
\hline \multirow[b]{2}{*}{ Trial } & \multicolumn{2}{|c|}{$\begin{array}{l}\text { No of patients with confirmed } \\
\text { myocardial infarction/ } \\
\text { no randomised }\end{array}$} & \multirow{2}{*}{$\begin{array}{l}\text { Observed } \\
\text { minus } \\
\text { expected }\end{array}$} & \multirow{2}{*}{ d Variance } \\
\hline & $\begin{array}{l}\text { Allocated to } \\
\text { calcium blocker }\end{array}$ & $\begin{array}{l}\text { Allocated to } \\
\text { control }\end{array}$ & & \\
\hline Bussman et al & $29 / 29$ & $25 / 25$ & 0.0 & 0.0 \\
\hline Crea $e t a l^{s}$ & $8 / 8$ & 9/9 & $0 \cdot 0$ & 0.0 \\
\hline Sirnes et $a l^{6}$ & $74 / 112$ & $83 / 115$ & $-3 \cdot 5$ & $12 \cdot 2$ \\
\hline Muller $e t a l^{\star \star}$ & $76 / 93$ & $73 / 88$ & -0.6 & 5.5 \\
\hline Gordon et $a l^{8}$ & $13 / 13$ & $13 / 13$ & 0.0 & 0.0 \\
\hline Gottlieb et $a l^{\circ}$ & $58 / 64$ & $55 / 68$ & $3 \cdot 2$ & $4 \cdot 1$ \\
\hline Branagan et al $l^{10}$ & $28 / 60$ & $23 / 61$ & $2 \cdot 7$ & $7 \cdot 4$ \\
\hline Loogna et a $l^{12}$ & $23 / 23$ & $34 / 34$ & 0.0 & 0.0 \\
\hline Wilcox et al ${ }^{\prime \prime}$ & $1429 / 2240$ & $1442 / 2251$ & $-3 \cdot 0$ & $259 \cdot 0$ \\
\hline Walker et al" & $146 / 217$ & $149 / 217$ & $-1 \cdot 5$ & $23 \cdot 7$ \\
\hline Jaffe $e t a l^{15}$ & $13 / 13$ & 9/9 & 0.0 & 0.0 \\
\hline Erbel $e t a l^{16}$ & $74 / 74$ & $75 / 75$ & 0.0 & 0.0 \\
\hline Zannad et $a l^{18}$ & $17 / 17$ & $16 / 17$ & 0.5 & $0 \cdot 3$ \\
\hline Machecourt et al $l^{19}$ & $34 / 38$ & $34 / 37$ & -0.5 & 1.6 \\
\hline Eichler $e t$ al $l^{20}$ & $16 / 16$ & $16 / 16$ & 0.0 & $0 \cdot 0$ \\
\hline Danish $^{\prime \prime} \dagger$ & $717 / 1729$ & $719 / 1718$ & $-3 \cdot 3$ & $209 \cdot 5$ \\
\hline SPRINT-II ${ }^{22}$ & $573 / 680$ & $589 / 678$ & -8.9 & $41 \cdot 6$ \\
\hline Total & $3328 / 5426(61 \cdot 3 \%)$ & $3364 / 5431(61.9 \%)$ & $-14 \cdot 9$ & 563.7 \\
\hline
\end{tabular}

*Additional data obtained through principal investigator.

^ $\$$ Additional data obtained through prin patients were entered $<24 \mathrm{~h}$.

†5\% Of patients were entered $<24 \mathrm{~h}$.
Overall typical odds ratio $(95 \%$ cond

Overall typical odds ratio ( $95 \%$ confidence interval) $0.97(0.90$ to 1.06$)$. No differences significant at $p \leqslant 0 \cdot 05$. Test for overall heterogeneity among all trials $\chi^{2} 7 \cdot 23,9 \mathrm{df}$

TABLE $\mathrm{v}-$ Release of serum enzyme in trials in which treatment was started within 24 hours

\begin{tabular}{|c|c|c|c|c|}
\hline \multirow[b]{2}{*}{ Trial } & \multirow[b]{2}{*}{ Enzyme measured } & No patients reported & $\begin{array}{l}\text { Measured enzyme } \\
\text { activity }\end{array}$ & \multirow[b]{2}{*}{ Ratio } \\
\hline & & $\begin{array}{l}\text { Calcium blocker/ } \\
\text { control }\end{array}$ & $\begin{array}{l}\text { Calcium blocker/ } \\
\text { control }\end{array}$ & \\
\hline Bussman et al & $\begin{array}{l}\text { Creatine kinase } M B \text { fraction } \\
\left(\mathrm{g} \mathrm{eq} / \mathrm{m}^{2}\right)\end{array}$ & $29 / 25$ & $31 / 49$ & $0.63^{\star}$ \\
\hline Crea $e t a l^{5}$ & $\mathrm{NA}$ & $0 / 0$ & $\begin{array}{l}\text { NA/NA } \\
\text { NA }\end{array}$ & NA \\
\hline Sirnes $e t a l^{\circ}$ & $\begin{array}{l}\text { Creatine kinase MB fraction } \\
\left(\mathrm{g} \text { eq } / \mathrm{m}^{2}\right)\end{array}$ & $71 / 77$ & $25 / 23$ & 1.09 \\
\hline Muller et a $l^{7}$ & $\begin{array}{l}\text { Creatine kinase MB fraction } \\
\left(\mathrm{g} \text { eq/ } \mathrm{m}^{2}\right)\end{array}$ & $68 / 65$ & $17 \cdot 0 / 16 \cdot 9$ & 1.01 \\
\hline Gordon et $a^{8}+$ & Creatine kinase (IU/l) & $13 / 13$ & NA & $1 \cdot 0$ \\
\hline Gottlieb et $a l^{\circ}$ & Creatine kinase (IU/1) & $64 / 68$ & $1383 / 1626$ & 0.85 \\
\hline Branagan et al" & $\begin{array}{l}\text { Creatine kinase } \mathrm{MB} \text { fraction } \\
(\mathrm{IU} / \mathrm{l})\end{array}$ & $23 / 17$ & $710 / 655$ & 1.08 \\
\hline Loogna et $a l^{12}$ & $\begin{array}{l}\text { Creatine kinase MB fraction } \\
(\mu \mathrm{kat})\end{array}$ & $23 / 34$ & $19 \cdot 4 / 19 \cdot 9$ & 0.97 \\
\hline Wilcox et $a l^{13}$ & NA & $0 / 0$ & NA/NA & NA \\
\hline Walker $e t a l^{l+}$ & $\begin{array}{l}\text { Creatine kinase } \mathrm{MB} \text { fraction } \\
\text { (IU/l) }\end{array}$ & $98 / 113$ & $406 / 345$ & $1 \cdot 08$ \\
\hline Jaffe $e t a l^{15}$ & $\begin{array}{l}\text { Creatine kinase } M B \text { fraction } \\
\left(\mathrm{g} \mathrm{eq} / \mathrm{m}^{2}\right)\end{array}$ & $9 / 8$ & $12 \cdot 6 / 9 \cdot 1$ & $1 \cdot 38$ \\
\hline Erbel et $a l^{16}$ & $\begin{array}{l}\text { Creatine kinase } M B \text { fraction } \\
\text { (IU/I) }\end{array}$ & $74 / 75$ & $680 / 590 \ddagger$ & $1 \cdot 15$ \\
\hline Zannad et al ${ }^{18}$ & $\begin{array}{l}\text { Creatine kinase MB fraction } \\
\text { (IU/1) }\end{array}$ & $13 / 14$ & $150 / 165$ & 0.91 \\
\hline Machecourt et al ${ }^{19}$ & Creatine kinase MB fraction & NA & NA & 0.865 \\
\hline $\begin{array}{l}\text { Danish }^{2134} \\
\text { SPRINT-II }\end{array}$ & Creatine kinase (IU/1) & $46 / 54$ & $3711 / 3436$ & 1.08 \\
\hline SPRINT-II ${ }^{22}$ & NA & $0 / 0$ & NA & $\mathrm{NA}$ \\
\hline
\end{tabular}

$\star$ Two tailed $\mathrm{p}$ value $<0.005$.

†Additional data obtained through principal investigator.

$\ddagger$ Approximate activites derived from graph.

\Reported as $-14 \%$.

The units used in various trials differ. No formal pooling has been attempted.

Enzyme activities were generally reported only in patients with a verified acute myocardial infarction (except for Gottlieb $\left.e t l^{*}\right)$. No trials, except when mentioned, significant at $p \leqslant 0 \cdot 05$.

$\mathrm{NA}=$ not available.

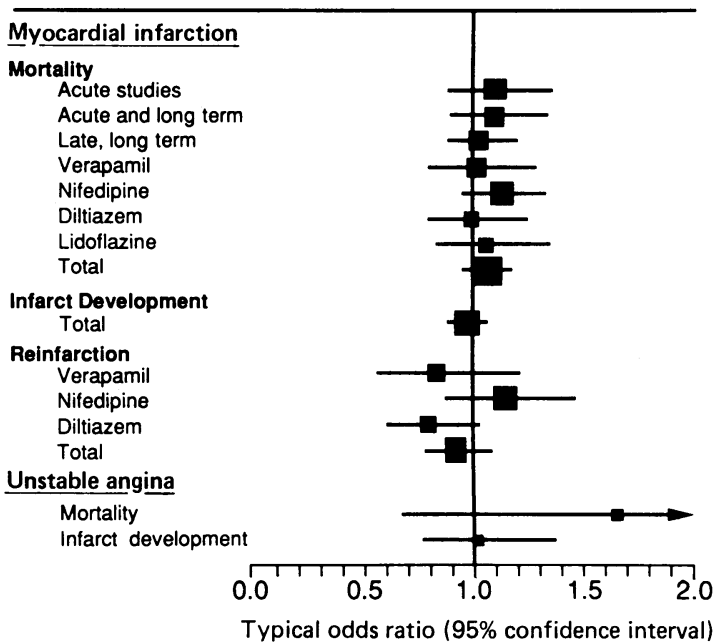

Typical odds of death, infarct development, and reinfarction by disease, types of trials, and drug used. Areas of squares proportional to number of patients. Bars indicate $95 \%$ confidence intervals. Portions to left of vertical line (corresponding to odds ratio $<1$ ) indicate reduced risk with treatment; portions to right of vertical line indicate increased risk with treatment. Upper $95 \%$ confidence limit for effect on mortality in unstable angina $6 \cdot 2$. Note that treatment does not seem to reduce risk of any event

$(61.9 \%)$ (overall typical odds reduction of $-3 \%$, $95 \%$ confidence interval $-10 \%$ to $6 \%$ ).

\section{Infarct size}

Data on enzymatically estimated size of infarct were available from 13 of the 16 trials in which treatment started within 24 hours (table V). Three trials reported only peak creatine kinase activities, while 10 trials measured peak or cumulative activities of the $\mathrm{MB}$ isomer of creatine kinase. Enzyme activities were, with one exception, ${ }^{9}$ reported only in patients developing a definite myocardial infarction. In most of the trials data were missing for several patients, probably because of difficulties in obtaining blood samples. In one large trial only a subset of the patients was studied..$^{21}{ }^{34}$ In five trials the enzyme release was lower in the treated group, in seven trials it was higher, and in one no difference was found. In one small open trial the difference was significant $(\mathrm{p}<0 \cdot 005){ }^{4}$

\section{Reinfarction}

Because of the difficulty in differentiating between an extension of the first infarction and reinfarction five early treatment trials with a study period of only 24-48 hours were excluded from this analysis. ${ }^{4111420}$ Reinfarction data were available from 11 of the 17 remaining trials for a total of 13900 patients (table VI, figure). In six trials the rate of reinfarction was lower in patients allocated to calcium channel blockers than in the control group and in four trials it was higher. One trial reported no reinfarctions in either group. ${ }^{19}$ In one trial the lower number of reinfarctions in the treated group almost reached nominal significance (two tailed $\mathrm{p}=0.06) .{ }^{17}$ Overall, 292 of 6939 patients $(4 \cdot 2 \%)$ allocated to calcium channel blockers suffered a nonfatal reinfarction compared with 317 of 6967 patients $(4.6 \%)$ in the control group (typical odds reduction of $9 \% ; 95 \%$ confidence interval $-24 \%$ to $7 \%$ ).

\section{Results by study drug}

Moderately large numbers' of patients have been studied with each drug except tiapamil. Table VII and the figure present the mortality data for each drug. No agent reduced mortality, and there was no statistical evidence of heterogeneity in the results among different agents.

Only with verapamil (three trials; 3518 patients) and nifedipine (10 trials; 7187 patients) were sufficient 
numbers of patients included early to examine the effect of treatment on development infarcts (tables I and IV, figure). With both these agents the proportion of patients developing myocardial infarction was similar in patients allocated to calcium channel blockers and controls (typical odds ratios $=0.99$ and $0 \cdot 98$, respectively).

Data on reinfarction were available for 3465 patients in trials of verapamil, for 7325 patients in trials of nifedipine, and 3117 patients in trials of diltiazem (table VII). Although there seemed to be a slight trend towards fewer reinfarctions in patients treated with verapamil and diltiazem, there was a non-significant increase compared with controls in the nifedipine trials. There was no statistical evidence of heterogeneity in the effects of these agents on reinfarctions, though the data were consistent with the possibility that agents that reduce heart rate (diltiazem and verapamil) might reduce the risk of reinfarction (167/ 3295 in the treated group compared with $208 / 3287$ in the control group; odds reduction of $20 \%$ ). This hypothesis is derived after viewing the data and should therefore be interpreted with caution and specifically examined in future trials.

\section{UNSTABLE ANGINA TRIALS}

Table II shows the results on development of myocardial infarction and deaths in the six trials of unstable angina. The largest trial was stopped early because of a trend towards more non-fatal myocardial

TABLE VI-Number of patients with non-fatal reinfarction in trials lasting over 48 hours

\begin{tabular}{|c|c|c|c|c|}
\hline \multirow[b]{2}{*}{ Trial } & \multicolumn{2}{|c|}{ No of reinfarctions/No randomised } & \multirow{2}{*}{$\begin{array}{l}\text { Observed } \\
\text { minus } \\
\text { expected }\end{array}$} & \multirow[b]{2}{*}{ Variance } \\
\hline & $\begin{array}{l}\text { Allocated to } \\
\text { calcium blocker }\end{array}$ & $\begin{array}{l}\text { Allocated to } \\
\text { control }\end{array}$ & & \\
\hline Crea et al & $4 / 8$ & $4 / 9$ & $0 \cdot 2$ & 0.9 \\
\hline Sirnes et al ${ }^{\circ}$ & $5 / 112$ & $5 / 115$ & $0 \cdot 1$ & $1 \cdot 3$ \\
\hline Muller $e t a l$ & NA & NA & NA & NA \\
\hline Gottlieb et $a l^{4}$ & $6 / 64$ & $7 / 68$ & -0.3 & 2.9 \\
\hline Eisenberg et $a l^{10 \star}$ & $2 / 25$ & $3 / 25$ & -0.5 & $1 \cdot 1$ \\
\hline Loogna et al ${ }^{12}$ & NA & NA & NA & NA \\
\hline Wilcox et $a l^{13}$ & $49 / 2240$ & $33 / 2251$ & $8 \cdot 1$ & $20 \cdot 1$ \\
\hline Jaffe et $a l^{\prime \prime}$ & NA & NA & NA & NA \\
\hline Erbel et al ${ }^{16}$ & $12 / 74$ & $8 / 75$ & $2 \cdot 1$ & $4 \cdot 4$ \\
\hline Gibson et al ${ }^{1}$ & $15 / 287$ & $27 / 289$ & $-5 \cdot 9$ & $9 \cdot 8 \dagger$ \\
\hline Zannad et al ${ }^{18}$ & NA & NA & NA & NA \\
\hline Machecourt et al $l^{19 \star}$ & $0 / 38$ & $0 / 37$ & $0 \cdot 0$ & $0 \cdot 0$ \\
\hline Danish $^{21} \ddagger$ & $50 / 1729$ & $60 / 1718$ & $-5 \cdot 2$ & $26 \cdot 6$ \\
\hline SPRINT-II ${ }^{22}$ & NA & NA & NA & NA \\
\hline MI Study Group ${ }^{23}$ & NA & NA & NA & NA \\
\hline SPRINT-I ${ }^{4}$ & $50 / 1130$ & $55 / 1146$ & $-2 \cdot 1$ & $25 \cdot 0$ \\
\hline MDPIT $^{2 s}$ & $99 / 1232$ & $116 / 1234$ & -8.0 & $48 \cdot 7$ \\
\hline Total & $292 / 6939(4 \cdot 2 \%)$ & $317 / 6967(4 \cdot 6 \%)$ & $-11 \cdot 5$ & $140 \cdot 8$ \\
\hline
\end{tabular}

^Additional information obtained through principal investigator †Two tailed $\mathrm{p}$ value $=0.06$

fOnly 1436 patients with confirmed myocardial infarction were treated beyond seven days. No data are available on remaining patients.

Overall typical odds ratio ( $95 \%$ confidence interval) $0.92(0.78$ to 1.09$)$ No results significant at $p \leqslant 0 \cdot 05$. Test for overall heterogeneity among all trials $\chi^{2} 9 \cdot 35,9 \mathrm{df}$.

$\mathrm{NA}=$ not available

TABLE VII - Mortality and non-fatal reinfarction by trial drug

\begin{tabular}{|c|c|c|c|c|c|}
\hline \multirow[b]{2}{*}{ Drug } & \multirow{2}{*}{$\begin{array}{c}\text { No } \\
\text { of } \\
\text { trials }\end{array}$} & \multicolumn{2}{|c|}{ No of events/No randomised } & \multirow{2}{*}{$\begin{array}{c}\text { Observed } \\
\text { minus } \\
\text { expected }\end{array}$} & \multirow[b]{2}{*}{ Variance } \\
\hline & & $\begin{array}{l}\text { Allocated to } \\
\text { calcium blocker }\end{array}$ & $\begin{array}{l}\text { Allocated to } \\
\text { control }\end{array}$ & & \\
\hline & & \multicolumn{2}{|c|}{ Mortality } &. & \\
\hline Verapamil & 3 & $149 / 1766(8 \cdot 4 \%)$ & $147 / 1752(8.4 \%)$ & $0 \cdot 6$ & $67 \cdot 7$ \\
\hline Nifedipine & 12 & $365 / 4731(7 \cdot 7 \%)$ & $330 / 4733(7 \cdot 0 \%)$ & $19 \cdot 3$ & $158 \cdot 8$ \\
\hline Diltiazem & 4 & $180 / 1574(11 \cdot 4 \%)$ & $181 / 1577(11.5 \%)$ & -0.4 & $78 \cdot 7$ \\
\hline Lidoflazine & 1 & $178 / 904(19 \cdot 7 \%)$ & $167 / 888(18.8 \%)$ & $4 \cdot 0$ & $69 \cdot 7$ \\
\hline Tiapamil & 1 & $0 / 16$ & $0 / 16$ & $0 \cdot 0$ & $0 \cdot 0$ \\
\hline \multicolumn{6}{|c|}{ Reinfarction } \\
\hline Verapamil & 2 & $54 / 1728(3 \cdot 1 \%)$ & $64 / 1727(3 \cdot 7 \%)$ & $-5 \cdot 0$ & $27 \cdot 5$ \\
\hline Nifedipine & 6 & $124 / 3645(3 \cdot 4 \%)$ & $111 / 3680(3 \cdot 0 \%)$ & $7 \cdot 4$ & $54 \cdot 8$ \\
\hline Diltiazem & 3 & $113 / 1557(7 \cdot 3 \%)$ & $142 / 1560(9 \cdot 1 \%)$ & $-13 \cdot 9$ & 58.5 \\
\hline
\end{tabular}

No results significant at $p \leqslant 0 \cdot 05$. Test for heterogeneity to assess differences between drugs for mortality $\chi^{2} 1 \cdot 1,3 \mathrm{df}$; for reinfarction $\chi^{2} 1 \cdot 7,2 \mathrm{df}$. infarctions in the subgroup that received only nifedipine. ${ }^{27}$ This was not observed when nifedipine was combined with metoprolol. Altogether the number of patients who developed myocardial infarction was 110 of 561 in patients allocated to calcium channel blockers compared with 104 of 591 in the control group; the corresponding number of deaths was 14 of 591 and nine of 578 , respectively.

\section{Discussion}

Calcium channel blockers are among the most commonly used drugs for patients with cardiovascular diseases. In 1987 the worldwide sales of these drugs accounted for about 19000 million Swiss francs, representing the largest expenditure for any class of cardiovascular drug (Q Hussain, personal communication). Calcium channel blockers are prescribed for several different indications, including hypertension, angina pectoris, acute myocardial infarction, and unstable angina. A recent survey showed that $42 \%$ of cardiologists in the United States would consider routinely using these drugs after uncomplicated myocardial infarction. . $^{35}$

Our overview of 19100 patients, based on all the available randomised trials in acute myocardial infarction and unstable angina, strongly suggests that the currently available calcium channel blockers are unlikely to reduce the rate of infarct development, infarct size, rate of reinfarction, or mortality. This result is not unexpected when separately viewing most trials; the overview additionally helped us to estimate the possible range of effects with higher precision. Although the confidence intervals do not exclude a modest benefit, a $10 \%$ reduction in mortality can probably be excluded. Moreover, the data suggest a somewhat higher probability of harm than benefit. The results are consistent when analysed by drug, by time of initiation of treatment, by duration of treatment, and by disease (unstable angina or myocardial infarction).

In some of the earlier postinfarction trials evaluating calcium channel blockers the lack of effect on mortality might have been due to the delay in giving the drug after the onset of ischaemia ${ }^{36}$ In animal experiments the most important anti-ischaemic effect has been found when these drugs are given before or soon after occlusion of the coronary artery. ${ }^{137}$ Data from the nifedipine trials of unstable angina do not, however, suggest a more beneficial clinical effect when the drug is given very early during ischaemia, indicating that the experimental data may not be directly relevant to the clinical situation. Although a small effect cannot be ruled out because of the small size of the unstable angina trials, the numbers of deaths and numbers of patients developing myocardial infarction were similar in the treated and control groups.

Even if calcium channel blockers do not reduce mortality a beneficial effect on morbidity could make preventive treatment worth while. The results are, however, equally disappointing when the effects of treatment on first or recurrent myocardial infarction and on enzyme release are considered. The lack of effect on infarct development, infarct size, and reinfarction reinforces the unpromising data on mortality. The data on infarct development were based on about 3500 patients in verapamil trials and 7200 patients in nifedipine trials but only about 100 in diltiazem trials. This makes the conclusions about diltiazem more uncertain. The available data on the effect of these drugs on infarct size do not even show a positive trend, an observation that makes large favourable effects unlikely. Furthermore, the pooled data on reinfarction also do not indicate any overall beneficial effect. Although the trend towards increased 
mortality in the calcium blocker group was found mainly in the nifedipine trials, no significant heterogeneity was seen in the results of different drugs on this or any of the other end points. As the power to detect interactions even in the pooled data is probably low only large interactions can be excluded.

Examination of the effect of treatment on several end points in a large number of trials may lead to spurious findings by chance, which may be nominally significant. Moreover, examination of the effects of treatment in multiple subgroups within each trial further increases the possibility of observing extreme results by chance. In several trials attempts have been made to identify subgroups in which treatment may have been harmful and others in which it may have been beneficial. In the multicentre diltiazem postinfarction trial a significant bidirectional interaction was claimed $-\mathbf{a}$ favourable but non-significant trend in those without pulmonary congestion (odds ratio 0.79 ; $95 \%$ confidence interval 0.57 to $1 \cdot 10$ )-whereas a "significant" adverse effect was observed in those with pulmonary congestion (odds ratio $1.85 ; 1.24$ to $2 \cdot 75){ }^{25}{ }^{38}$ As stated by the authors, these analyses should be cautiously interpreted because they have not yet been verified in a prospective study. Furthermore, most of the remaining trials excluded patients with pulmonary congestion or heart failure (table I) and yet observed a trend towards increased mortality.

In the short term trial by Gibson et al randomising only patients with non- $Q$ wave myocardial infarction, $a$ lower number of reinfarctions after 14 days of treatment was found in patients taking diltiazem than in the control group. ${ }^{17}$ The results in this trial reached conventional levels of significance $(p \leqslant 0.05)$ on a one tailed test but not on a two tailed test. Data on non- $Q$ wave myocardial infarction were available from only two other studies. ${ }^{1025}$ In the study by Eisenberg et al two of 25 patients taking nifedipine compared with three of 25 control patients developed a reinfarction. ${ }^{10}$ In the long term multicentre diltiazem postinfarction trial $9 \%$ of 296 patients treated with diltiazem compared with $15 \%$ of 338 control patients developed a reinfarction or suffered a cardiac death at the end of one year of treatment. ${ }^{25}$ Data from this subgroup were not available for the full length of the trial ( 25 months' average follow up). The data on non- $Q$ wave myocardial infarction are thus incomplete even for the diltiazem trials. If these data are promising separate analyses of patients with non- $Q$ wave myocardial infarction in all completed trials may be warranted and perhaps prospective evaluation in large trials. Therefore, there is no conclusive evidence that calcium channel blockers reduce mortality or reinfarction in any particular subgroup of patients.

This overview of all available randomised trials of calcium channel blockers in myocardial infarction and unstable angina does not indicate any beneficial effect on infarct development, infarct size, reinfarction, or mortality. At present calcium blockers cannot be recommended prophylactically in patients during or after acute myocardial infarction or in those with unstable angina.

We thank Drs W-D Bussman, H De Geest, P Eisenberg, J Lubsen, I Machecourt, A Moss, L Opie, B Rosner, J Tijssen, $\mathrm{L}$ Walker, $M$ Walsh, $\mathrm{R}$ Wilcox, and $\mathrm{F}$ Zannad for providing additional data from their trials; Dr R Collins for advice; Dr Q Hussain for information on sales of calcium antagonists; and Ms Zola Hall for typing this manuscript.

1 Kloner RA, Braunwald E. Effects of calcium antagonists on infarcting myocardium. Am $\mathcal{f}$ Cardiol 1987;59:84-94B.

2 Opie LE, Buhler FR, Fleckenstein A, et al. Working group on classification of calcium antagonists for cardiovascular disease. Am 7 Cardiol 1987;60:630-2.

3 Yusuf S, Peto R, Lewis J, Collins R, Sleight P. Beta blockade during and after myocardial infarction: an overview of the randomized trials. Prog Cardiovasc Dis 1985;17:335-71.
4 Bussman WD, Ser W, Gruengrass $M$. Reduction of creatine kinase and creatine kinase-MB indexes of infarct size by intravenous verapamil. Am f Cardiol 1984;54:1224-30.

5 Crea F, Deanfield J, Crean P, Sharom M, Davies G, Maseri A. Effects of verapamil in preventing early post-infarction angina and reinfarction. Am ₹ Cardiol 1985;55:900-4.

6 Sirnes PA, Overskeid K, Pedersen TR, et al. Evaluation of infarct size during the early use of nifedipine in patients with acute myocardial infarction. The Norwegian nifedipine multicenter trial. Circulation 1984;70:638-44.

7 Muller JE, Morrison J, Stone $\mathrm{PH}$, et al. Nifedipine therapy for patients with threatened and acute myocardial infarction: a randomized, double-blind, placebo-controlled comparison. Circulation 1984;69:740-7.

8 Gordon GD, Mabin TA, Isaacs S, Lloyd EA, Eichler HG, Opie LH Hemodynamic effects of sublingual nifedipine in acute myocardial infarction. Am $\mathcal{F}$ Cardiol 1984;53:1228-32.

9 Gottlieb SO, Becker L, Weiss JL, et al. Nifedipine in acute myocardial infarction: an assessment of left ventricular function, infarct size, and infarct expansion a double-blind randomized trial. $B r$ Heart $f$ 1988;59:411-8.

10 Eisenberg PR, Lee RG, Biello DR, Geltman EM, Jaffe AS. Chest pain after nontransmural infarction: the absence of remediable coronary vasospasm. Am Heart f 1985;110:515-21.

11 Branagan JP, Walsh K, Kelly P, Collins WC, McCafferty D, Walsh MJ. Effect of early treatment with nifedipine in suspected acute myocardial infarction. Eur Heart $f$ 1986;7:859-65.

12 Loogna E, Sylven C, Groth T, Mogensen L. Complexity of enzyme release during acute myocardial infarction in a controlled study with early nifedipine treatment. Eur Heart $f$ 1985;6:114-9.

13 Wilcox RG, Hampton JR, Banks DC, et al. Trial of early nifedipine in acute myocardial infarction: the TRENT study. Br Med f 1986;293:1204-8.

14 Walker L, Mackenzie A, Adgey J. Effect of nifedipine on enzymatically estimated infarct size in the early phase of acute myocardial infarction. Br Heart ₹ 1988;39:403-10.

15 Jaffe AS, Biello DR, Sobel BE, Geltman EM. Enhancement of metabolism of jeopardized myocardium by nifedipine. Int $\mathcal{F}$ Cardiol 1987;15:77-89.

16 Erbel R, Pop T, Meinertz T, et al. Combination of calcium channel blocker and thrombolytic therapy in acute myocardial infarction. Am Hean $f$ 1988;115:529-38

17 Gibson RS, Boden WE, Theroux P, et al. Diltiazem and reinfarction in patients with non-Q wave myocardial infarction. $N$ Engl f Med 1986;315: 423-9.

18 Zannad F, Amor M, Karcher G, et al. Effect of diltiazem on infarct size estimated by enzyme release, serial thallium-201 single photon emission computed tomography and radio nuclide angiography. Am f Cardiol 1988;61:1172-8.

19 Machecourt J, Cassagnes J, Andre-Fouet X, et al. Diltiazem infused within 6 hours after an acute anterior myocardial infarction in man. Significan reduction of ventricular arrhythmias; no significant reduction of infarct size. In: Proceedings of $X$ world congress of cardiology. Washington, DC: American Heart Association, 1986:250A.

20 Eichler HG, Mabin TA, Commerford PJ, Lloyd EA, Beck W, Opie LH Tiapamil, a new calcium antagonist:hemodynamic effects in patients with acute myocardial infarction. Circulation 1985;71:779-86.

21 Danish Study Group on Verapamil in Myocardial Infarction. Verapamil in acute myocardial infarction. Eur Heart f 1984;5;516-28.

22 SPRINT Study Group. The secondary prevention re-infarction Israeli nifedipine trial (SPRINT) II: design and methods, results. Eur Heart $\mathcal{f}$ 1988;9(suppl 1):350A.

23 Myocardial Infarction Study Group. Secondary prevention of ischemic hear disease: a long term controlled lidoflazine study. Acta Cardiol 1979, 34(suppl 24):7-46.

24 The Israeli SPRINT Study Group. Secondary prevention reinfarction Israel nifedipine trial (SPRINT). A randomized intervention trial of nifedipine in patients with acute myocardial infarction. Eur Hean $\mathcal{F}$ 1988;9:354-64.

25 Multicenter Diltiazem Postinfarction Trial Research Group. The effect of diltiazem on mortality and reinfarction after myocardial infarction. $N E n g l f$ Med 1988;319:385-92.

26 Gerstenblith G, Ouyang P, Achuff SC, et al. Nifedipine in unstable angina. A double-blind, randomized trial. $N$ Engl F Med 1982;306:885-9.

27 Holland Interuniversity Nifedipine/Metoprolol Trial (HINT) Research Group. Early treatment of unstable angina in the coronary care unit: randomized, double-blind, placebo controlled comparison of recurren ischemia in patients treated with nifedipine or metoprolol or both. Br Heart f 1986;56:400-13.

28 Hagemeijer F, Schelling A, Marinissen K, Van MR. Impending myocardial infarction treated intravenously with diltiazem. In: Proceedings of infarction treated intravenously with diltiazem. In: Proce
cardiovascular pharmacotherapy symposium. Geneva: 1985:314A.

29 Muller JE, Turi ZG, Pearle DL, et al. Nifedipine and conventional therapy for unstable angina pectoris: a randomized, double blind comparison. Circulation 1984;69:728-39.

30 Theroux P, Taeymans Y, Morrisette D, Bosch X, Pelletier GB, Waters D. A randomized study comparing propranolol and diltiazem in the treatment of unstable angina. $\mathcal{F}$ Am Coll Cardiol 1985;5:717-22.

31 André-Fouet X, Usdin JP, Gayet C, et al. Comparison of short-term efficiacy of diltiazem and propranolol in unstable angina at rest. A randomized trial in 70 patients. Eur Heart $\mathcal{F}$ 1983;4:691-8.

32 Parodi O, Maseri A, Simonetti I. Management of unstable angina at rest by verapamil. A double-blind cross-over study in coronary care unit. Br Heart $\mathcal{f}$ 1979;41:167-74.

33 Mehta J, Pepine CJ, Day M, Guerrero JR, Conti CR. Short-term efficacy of oral verapamil in rest angina. $A m \mathcal{F}$ Med 1981;71:977-82.

34 Thuesen L, Jorgensen JR, Kvistgaard HJ, et al. Effects of verapamil on enzyme release after early intravenous administration in acute myocardial enzyme release after early intravenous administration in acute myoc

35 Hlatky MA, Cotugno HE, Mark DB, O'Connor C, Califf RM, Pryor DB. Trends in physician management of uncomplicated acute myocardial Trends in physician management of uncomplicated
infarction, 1970 to 1987. Am $\mathcal{f}$ Cardiol 1988;61:515-8.

36 Hugenholtz PG, Serruys PW, Fleckenstein A, Nayler W. Why $\mathrm{Ca}^{2+}$ antagonists will be most useful before or during early myocardial ischemia and no after infarction has been established. Eur Heart $\mathcal{f} 1986 ; 7: 270-8$.

37 Reimer KA, Jennings RB. Effects of calcium-channel blockers on myocardia preservation during experimental acute myocardial infarction. Am $\mathcal{F}$ Cardio 1985;55:107-15B.

38 Moss A, Oakes D, Benhorin J. Effect of diltiazem on outcome in postinfarction patients with and without left ventricular dysfunction. Circulation 1988;78 (suppl II):97A.

(Accepted 30 August 1989) 